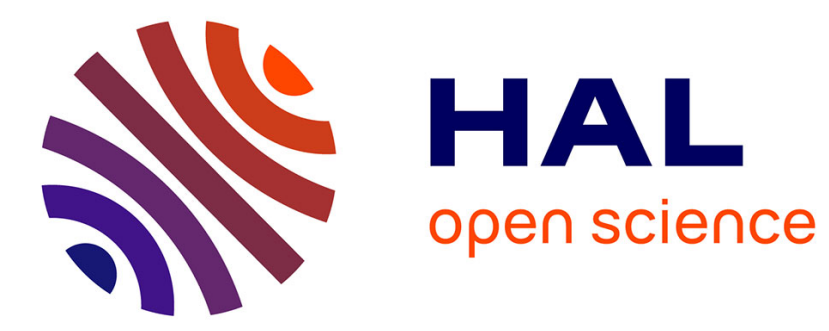

\title{
Computer-controlled high-precision Michelson wavemeter
}

\author{
J Pedregosa-Gutierrez, D Guyomarc'h, M Vedel, C Champenois, Martina \\ Knoop
}

\section{- To cite this version:}

J Pedregosa-Gutierrez, D Guyomarc'h, M Vedel, C Champenois, Martina Knoop. Computercontrolled high-precision Michelson wavemeter. The European Physical Journal Plus, 2014, 129 (9), pp.203. 10.1140/epjp/i2014-14203-3 . hal-01455333

\section{HAL Id: hal-01455333 https://hal.science/hal-01455333}

Submitted on 19 Apr 2019

HAL is a multi-disciplinary open access archive for the deposit and dissemination of scientific research documents, whether they are published or not. The documents may come from teaching and research institutions in France or abroad, or from public or private research centers.
L'archive ouverte pluridisciplinaire HAL, est destinée au dépôt et à la diffusion de documents scientifiques de niveau recherche, publiés ou non, émanant des établissements d'enseignement et de recherche français ou étrangers, des laboratoires publics ou privés. 


\title{
Computer-controlled high-precision Michelson wavemeter
}

\author{
J. Pedregosa-Gutierrez, ${ }^{*}$ D. Guyomarc'h, M. Vedel, C. Champenois, and M. Knoop \\ Aix-Marseille Université, CNRS, PIIM, UMR 7345, \\ Centre de Saint Jérôme, Case C21, 13397 Marseille Cedex 20, France
}

(Dated: July 17, 2014)

\begin{abstract}
The Michelson wavemeter is a popular instrument in many experiments where the high precision measurement of a $\mathrm{cw}$ laser wavelength is required. In this paper, we describe a simple and inexpensive way to obtain high precision measurements with this classical physicist's tool. We exploit the time stamp provided by the high frequency clock present in modern data acquisition cards to measure the fractional uncertainty of the interference signal. The resulting relative uncertainty value for our current set-up is of the order of $10^{-8}$ and can be potentially improved by a factor of 100 .
\end{abstract}

\section{INTRODUCTION}

The accurate measurement of a laser wavelength is an important step in many situations in experimental physics involving resonant excitation of an atomic transition or an optical resonator, for example in laser cooling of a trapped sample as in our experiments [3]. To this purpose, the laser wavelength has to be adjusted within the linewidth of the excited atomic transition, with typical values of the order of $\Delta \nu_{F W H M} \approx 20 \mathrm{MHz}$. A widely used instrument to obtain such precision measurements is the scanning Michelson wavemeter, first introduced in $1976[6,9]$. Using this type of wavemeter, it is possible to achieve accuracies of a few parts in $10^{9}$ [13]. However, these high accuracies are at the expenses of a complex set-up.

In the following, we propose an alternative to the technique used by Bennett and Gill in [1] which takes advantage of modern data acquisition cards to achieve a calculated wavelength uncertainty below $2 \cdot 10^{-8}$ by using a conventional set-up.

\section{PRINCIPLE OF OPERATION OF A WAVEMETER}

A Michelson wavemeter is based on a travelling Michelson interferometer and is described in detail in many text-

*jofre.pedregosa@univ-amu.fr

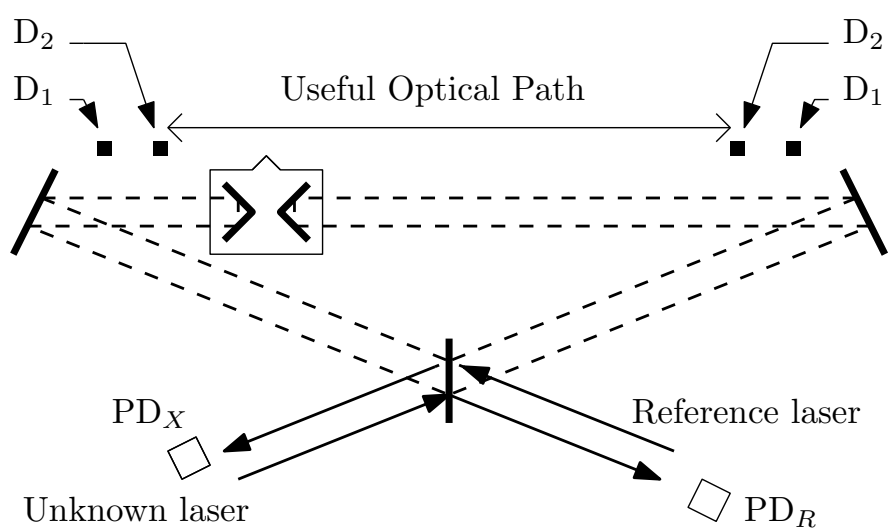

FIG. 1: Principle of a Michelson wavemeter. Two laser beams, the reference and the unknown one, are injected into a travelling Michelson interferometer. The interference patterns are read out by two photo-detectors $\left(\mathrm{PD}_{X}\right.$ and $\left.\mathrm{PD}_{R}\right)$, amplified and sent to a counter. Detectors labelled as $D_{1}$ are used to change the direction of the cubes's carrier, while the detectors

$D_{2}$ define the useful travelling path by gating the measurement sequence.

books, see for example [5]. Therefore only a brief review is presented here. The set-up considered (see figure 1) involves two corner cubes mounted on the same carriage. The translation of this carriage induces a varying path difference between each arm of the interferometer. Two laser beams, coming from a reference laser $(R)$ and a laser of unknown wavelength $(X)$ go through the interferometer on identical paths but opposite direction. The resulting interference patterns are measured by photodiodes, amplified, and sent to a counter. In such a set-up the 
ratio of the laser wavelengths is inversely proportional to the ratio of the number of interference fringes measured for each laser.

Each interference signal is modulated as:

$$
S(t)=A \cos \left(\frac{8 \pi v t}{\lambda_{i}}+\phi\right)
$$

where $v$ is the speed of the carrier, and $\lambda_{i}$ the laser wavelength. The number of maxima, $n_{i}$, for a given path length $L$ of the carriage, is:

$$
2 \pi\left(n_{i}+\epsilon\right)=\frac{8 \pi L}{\lambda_{i}}+\phi
$$

where $\epsilon$ represents the fact that the path length $L$ is not an integer multiple of the laser half-wavelength. The phase $\phi$ takes into account the fact that the data acquisition is not necessarily started in phase with the interference pattern. These two quantities, $\epsilon$ and $\phi$ both represent the mismatch of the path length with the interference pattern. They are kept separate as $\phi$ represents a mismatch at the beginning while $\epsilon$ happens at the end of the measurement period, and therefore the technical solutions to reduce them to zero are different.

The wavelength values in equation 2 are taken in air and therefore the variation of the refractive index of air with the wavelength is not taken into account. The error introduced by this assumption will be discussed later.

By applying equation 2 to the reference laser $\left(\lambda_{R}\right)$ and the unknown one $\left(\lambda_{X}\right)$, we obtain:

$$
\lambda_{X}=\lambda_{R} \frac{n_{R}+\epsilon_{R}-\frac{\phi_{R}}{2 \pi}}{n_{X}+\epsilon_{X}-\frac{\phi_{X}}{2 \pi}}
$$

It is possible, with the adequate electronics, to trigger the counter with one of the interference signals, effectively making $\phi_{R}=0$. Additionally, the electronics can be configured to count an exact number of maxima, which means that $\epsilon_{R}=0$. Therefore, equation 3 can be simplified to:

$$
\frac{\lambda_{X}}{\lambda_{R}}=\frac{n_{R}}{n_{X}+\epsilon_{X}-\frac{\phi_{X}}{2 \pi}}
$$

The term $\epsilon_{X}-\frac{\phi_{X}}{2 \pi}$ is often neglected due to the difficulty of measuring $\phi_{X}$ and $\epsilon_{X}$. The equation used to compute the unknown $\lambda_{X}$ is then reduced to

$$
\lambda_{X}=\lambda_{R} \frac{n_{R}}{n_{X}}
$$

and the error $\delta \lambda_{X}$ due to the neglected term is bounded by:

$$
\frac{\delta \lambda_{X}}{\lambda_{X}} \leq \frac{2}{n_{X}}
$$

where we have used that $0<\left|\epsilon-\frac{\phi}{2 \pi}\right|<2$.

By using this technique, a typical set-up with a travelling distance of $0.5 \mathrm{~m}$, using a He-Ne laser as reference source to measure $\lambda=845.3460 \mathrm{~nm}$, (which correspond to a given atomic transition in our set-up) gives an error of $\delta \lambda_{X} \approx 8 \cdot 10^{-4} \mathrm{~nm}$ corresponding to a frequency uncertainty $\Delta \nu_{X} \approx 300 \mathrm{MHz}$. This value is far above the FWHM of the considered atomic transition. For many atomic physics experiments, this resolution is not sufficient to tune an exciting laser to a resonant line.

A standard way to improve the precision of the measurement is to increase $n_{X}$ when using the approximation given by eq. 5 , as the other parameters are fixed $\left(\lambda_{R}, \lambda_{X}\right)$ or bounded $\left(\phi_{R}, \epsilon_{R}\right)$. The straightforward approach is to increment the travelling path length. This can be achieved in a compact manner as shown in [10]. Alternatively, it is possible to multiply the $n_{X}$ counts by a constant factor using a Phase Locked Loop, as it was first demonstrated by [6]. The first solution involves a redesign/modification of the mechanical set-up. The second approach involves relatively advanced electronics if one aims to obtain a precise measurement with a rate of one per second.

Another possibility is to bring down to zero $\epsilon_{R}$, $\phi_{R}, \epsilon_{X}$ and $\phi_{X}$ simultaneously using the coincidence method $[7,8]$. This consists in starting and stopping the measurement at an instant when the phases of both lasers are equal. When the interval is specified in this manner, the precision of the frequency measurement is limited by the temporal resolution of the electronics which detects the phase matching.

Alternatively, $\epsilon_{R}$ and $\phi_{R}$ can also be determined. This has been realized in [12] by using a least-squares fit to the data. The implementation presented in [12] requires to store the produced interference fringes, with a good enough sampling of the signal for the curve fitting to be relevant.

A more accurate way to measure $\epsilon_{R}$ and $\phi_{R}$ has been given by Bennett and Gill [1]. From the electronics point of view, the implementation presented in [1] requires 
advanced custom-made electronics.

In this manuscript, we show how this last type of measurements can be easily realized using commercial acquisition cards. These cards are commonly used in industry and therefore are affordable and very well documented, simplifying their practical implementation. Moreover, we present an improved algorithm that allows a factor 2 gain in uncertainty respect [1]. Experimental results of this new technique are also provided and discussed.

\section{TIME STAMP APPROACH FOR HIGH RESOLUTION MEASUREMENTS}

\section{A. Numerical implementation of Bennett's method}

The principle of this method is illustrated in figure 2, showing the two interference patterns after being transformed to TTL pulses and the gate signal used to start and stop the actual data acquisition. Using the time intervals $a, b, c$ and $d$ defined on figure 2 , it is possible to obtain $\epsilon_{X}$ and $\phi_{X}$ by [1]

$$
\epsilon_{X}-\frac{\phi_{X}}{2 \pi}=\frac{a}{b}+\frac{d}{c}
$$

This equation assumes that the velocity of the carrier remains constant only during the measurement of $a$ and $b$ and of $c$ and $d$. This eliminates the need for highly stabilized velocities during the complete measurement, a requirement which is often found for other methods proposed in the literature. The unknown wavelength, $\lambda_{X}$, is obtained using:

$$
\lambda_{X}=\lambda_{R} \frac{n_{R}}{n_{X}+\frac{a}{b}+\frac{d}{c}}
$$

\section{B. Improved method}

The time-stamp approach needs less measurements. As indicated in figure 2, only four times need to be measured, instead of six in the method in III A. The total measuring time, $t_{m}$, can be written as a function of the average period of each interference pattern, $\left\langle\tau_{i}\right\rangle$ :

$$
\begin{aligned}
t_{m} & =t_{4}-t_{1}=n_{R}<\tau_{R}> \\
t_{m} & =n_{X} \tau_{X}+\left(t_{4}-t_{3}\right)+\left(t_{2}-t_{1}\right)=n_{X}<\tau_{X}>+t^{\prime}
\end{aligned}
$$

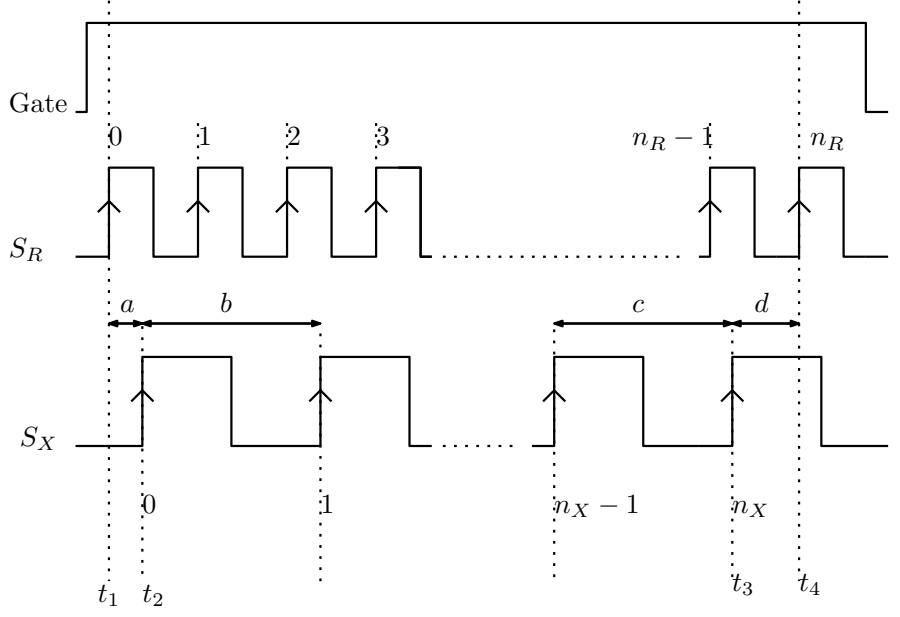

FIG. 2: Diagram showing the different signals involved in the measurement, together with the different times and times intervals which need to be exactly measured for a precise final wavelength determination.

The reason to use average values for $\tau$, is that the velocity may not be constant during the measuring time, $t_{m}$. Assuming the wavelengths are fixed, we can rewrite equation 9 as:

$$
\begin{aligned}
& <\tau_{R}>=\frac{t_{m}}{n_{R}}=\frac{\lambda_{R}}{4<v_{1}>} \\
& <\tau_{X}>=\frac{t_{m}-t^{\prime}}{n_{X}}=\frac{\lambda_{X}}{4<v_{2}>}
\end{aligned}
$$

where $\left\langle v_{1}\right\rangle$ is averaged over $t_{m}$ while $\left\langle v_{2}\right\rangle$ is averaged over $t_{m}-t^{\prime}$. A possible difference could be introduced during the extra time, $t^{\prime}$. As $t^{\prime} / t_{m}$ is of the order of $10^{-6}$, we can safely assume that $\left\langle v_{1}\right\rangle=\left\langle v_{2}\right\rangle$ in that extra time $t^{\prime}$. This assumption is equivalent to the one made by Bennett and Gill, where the velocity is assumed constant over two consecutive maxima at the start and at the end of the carriage travelling.

By replacing $\left\langle\tau_{X}>\right.$ and $\left\langle\tau_{R}>\right.$, we obtain the final expression:

$$
\lambda_{X}=\lambda_{R} \frac{n_{R}}{n_{X}}\left(1-\frac{t^{\prime}}{t_{m}}\right)
$$

There is a non-negligible advantage of using the method described by equation (11) rather than equation (8), as it becomes clear from the error budgets, see section IIID. 


\section{Technical implementation}

The implementation of the proposed improved scheme is made by a National Instrument PCIe-6363 acquisition card. The counter associated to the card's internal clock is started by the gate signal. Each incoming TTL pulse generates a new register entry (stored in the card's internal buffer), recording the number of clock cycles elapsed since the start of the acquisition, see figure 3 . This value is referred to as the time stamp. The result is a vector of variable size, whose dimension is the number of incoming pulses, and whose contents are the time stamps of each incoming pulse. The size of the two vectors created in this way, will therefore give the values of $n_{X}$ and $n_{R}$. The contents are the time intervals in units of the acquisition card clock's period, giving all the information needed to compute eq. 8 or eq. 11 .

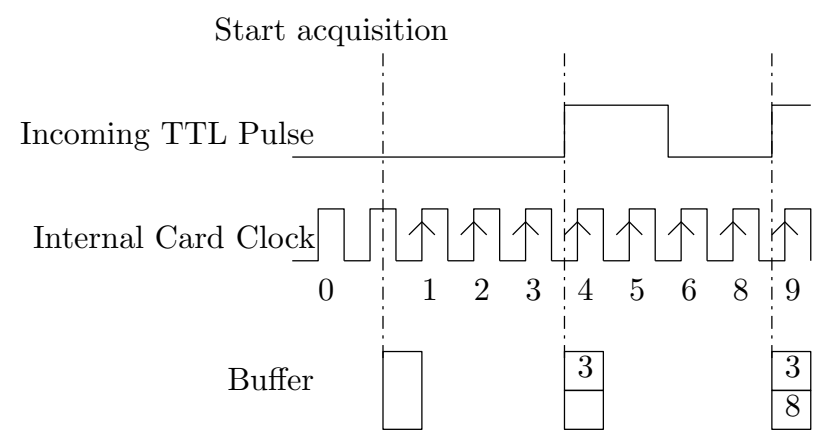

FIG. 3: For each interference signal, the acquisition card saves in a buffer the time stamp for every incoming TTL pulse ( $S_{R}$ and $S_{X}$ signals). In this example, two rising edges are recorded with a time difference of 5 clock periods between both pulses.

\section{Error budgets}

To compare error budgets of the two presented methods, common relevant values for typical wavemeters have been used: a travelling path of $40 \mathrm{~cm}$, travelled in $1 \mathrm{~s}$. This implies for our unknown wavelength $\lambda_{X}=845.3 \mathrm{~nm}$ an average interference signal period $\left\langle\tau_{X}\right\rangle=0.5 \mu$ s and a total number of fringes $n_{X}$ of $2.10^{6}$. If $\Delta t$ is the error on one time interval measurement, the maximum relative uncertainty of the measurement using Bennett's method (eq. 8), can be written as:

$\left|\frac{\Delta \lambda_{X}}{\lambda_{X}}\right|_{B} \leq\left|\frac{\Delta t}{n_{X}}\left(\frac{1}{b}+\frac{a}{b^{2}}+\frac{1}{c}+\frac{d}{c^{2}}\right)\right|+\left|\frac{\Delta \lambda_{R}}{\lambda_{R}}\right|+\left|\frac{\Delta r}{r}\right|+\left|\frac{\delta s}{\Delta s}\right|$

where the last two terms are taking into account the dependency on the refraction index and misalignments [5].

Let us estimate the magnitude of each term in equation (12). The first term corresponds to the sum of errors associated to the different time intervals. As each time interval is determined by a subtraction of two time stamps, and each time stamp has an error of half the clock period, $\tau_{\text {card }}$, we obtain $\Delta t=\tau_{\text {card }}$. By using a $100 \mathrm{MHz}$ internal clock, the value of $\tau_{\text {card }}$ is $10 \mathrm{~ns}$. As $a / b$ and $d / c \leq 1$ and $b$ and $c$ are of the order of $\left\langle\tau_{X}\right\rangle$, this term is bounded by $4 \tau_{\text {card }} /\left(<\tau_{X}>n_{X}\right)=4 \cdot 10^{-8}$.

The second term of equation (12) depends on the reference laser. A commercial, temperature-stabilized He-Ne laser has a nominal frequency stability of $2 \mathrm{MHz}$, leading to $\left|\frac{\Delta \lambda_{R}}{\lambda_{R}}\right| \approx 4 \cdot 10^{-9}$.

The third term reflects the dependency of the index of refraction of air, $n_{0}$, on the wavelength. It can be estimated using [5]:

$$
\left|\frac{\Delta r}{r}\right| \approx 1 \cdot 10^{-3}\left|n_{0}\left(\lambda_{X}\right)-n_{0}\left(\lambda_{R}\right)\right|
$$

A value of $\lambda_{X}=845.3460 \mathrm{~nm}$ leads to $\left|\frac{\Delta r}{r}\right| \approx 2 \cdot 10^{-9}$.

A possible misalignment of the laser beams resulting in signal variations is taken into account by the fourth term, where $\Delta s$ is the optical path difference for each arm of the interferometer and $\delta s=\Delta s\left(\lambda_{R}\right)-\Delta s\left(\lambda_{X}\right)$. To keep the introduced systematic relative error $\left|\frac{\delta s}{\Delta s}\right|$ lower than $10^{-8}$, the tilt angle between the two beams must be smaller than $2 \cdot 10^{-4} \mathrm{rad}$ [5]. Let's point out that, for a given alignment, this is a systematic error affecting the accuracy but not the precision of the measurement. With those values, the estimated maximum relative uncertainty of $\lambda_{X}=845 \mathrm{~nm}$ is $4 \cdot 10^{-8}$, corresponding to a frequency uncertainty $\Delta \nu_{X}=14 \mathrm{MHz}$.

The proposed improved method relies on the measurement of the full measurement time $t_{m}$. The extra time $t^{\prime}$ can not be longer than 2 interference signal periods 
$<\tau_{X}>$. It induces that $t^{\prime} / t_{m} \leq 10^{-6}$ and the maximum relative uncertainty of the $\lambda_{X}$, expressed by eq. 11 is given by:

$$
\left|\frac{\Delta}{\lambda_{X}}\right| \leq\left|\frac{\Delta t_{m} t^{\prime}}{t_{m}^{2}}\right|+\left|\frac{\Delta t^{\prime}}{t_{m}}\right|+\left|\frac{\Delta \lambda_{R}}{\lambda_{R}}\right|+\left|\frac{\Delta r}{r}\right|+\left|\frac{\delta s}{\Delta s}\right|
$$

where the last three terms have the same meaning as in equation (12), while the first two terms describe uncertainties due to the technical implementation of the method. The first term is due to the error on the total measuring time. It is determined by the on-board clock of the acquisition card. Our card has an internal $100 \mathrm{MHz}$ clock with a nominal uncertainty of $50 \mathrm{ppm}$. This uncertainty is the dominating term for acquisition times much longer than the clock period. For the chosen measurement time of $1 \mathrm{~s}$, we can consider $\Delta t_{m} \approx 50 \mu \mathrm{s}$. Furthermore, the extra time $t^{\prime}$ is the sum of two measured intervals whose duration is of the order of $\left\langle\tau_{X}>\right.$ leading to $\left|\frac{\Delta t_{m} t^{\prime}}{t_{m}^{2}}\right| \leq\left|\frac{\Delta t_{m} 2<\tau_{X}>}{t_{m}^{2}}\right| \approx 5 \cdot 10^{-11}$.

The second term arises from the measurement uncertainty $\Delta t^{\prime}$ of the two intervals $\left(t_{4}-t_{3}\right)$ and $\left(t_{2}-t_{1}\right)$ which are similar to the $a, b, c$ and $d$ intervals of eq. 12 . The uncertainty now counts twice instead of 4 times and $\Delta t^{\prime}$ is then equal to $2 \tau_{\text {card }}$ and $\left|\frac{\Delta t^{\prime}}{t_{m}}\right|=\frac{2 \tau_{\text {card }}}{t_{m}}=2 \cdot 10^{-8}$. The total estimated maximum relative uncertainty for the digital implementation is dominated by this contribution. For this method, the uncertainty related to the measurement of the full time $t_{m}$ is negligible compared to the contribution of the extra time, $t^{\prime}$, measurement.

As the term $\left\langle\tau_{X}>n_{X}\right.$ is nearly equal to $t_{m}$, for both methods presented above, the leading term for measurement uncertainty scales like $\tau_{c a r d} / t_{m}$, with a factor 2 instead of 4 in the improved proposed method because only 2 intervals are needed instead of 4 . The acquisition card technology limits $\tau_{\text {card }}$ and it may be improved in the future. The precision of the measurement can be easily increased by taking a longer measurement time $t_{m}$. Note that the budget error given by eq. 12 does not depend on the total $t_{m}$, but on $n_{x}$, which depends only on the length $\mathrm{L}$ and not on the speed of the carrier. For $t_{m}$ larger than $100 \mathrm{~s}$, the uncertainty budget is limited by other contributions. To improve the error arising from the reference laser it is possible to use one of the several existing techniques to improve the stability of a laser [4]. Indeed, with a highly-stabilized $100 \mathrm{kHz}$ He-Ne reference laser, $\left|\frac{\Delta \lambda_{R}}{\lambda_{R}}\right|$ is reduced to $<10^{-10}$. Regarding, the refractive index of air, several set-ups [2] exist where the wavemeter is under vacuum, making the corresponding term in the total error budget negligible. Furthermore, advanced techniques, as shown in [12], allow to reduce the systematic error induced by misalignment. Therefore, in a system making use of the various cited techniques, it is possible to imagine a motorized set-up that can be operated in a "low" precision measurements $\left(\Delta \nu / \nu \approx 2 \cdot 10^{-8}\right)$ every second, and, if needed, with a simple switch, in a "high" precision measurement $\left(\Delta \nu / \nu \approx 2 \cdot 10^{-10}\right)$ by decreasing the carrier's speed by a factor of 100 .

\section{EXPERIMENTAL IMPLEMENTATION}

The main advantage of the proposed method is the small number of modifications that an exisiting travelling Michelson wavemeter set-up needs. In our particular case, we had an existing implementation with the carriage being pulled back and forth by a DC motor. The carrier moves on a V-rail using an air flow to minimize friction. Two photo-detectors sensitive to the passage of the carriage at each end of the rail inverse the sense of the motor $\left(D_{1}\right.$ on figure 1$)$. To make sure to count fringes when the carriage velocity is nearly uniform, two additional detectors $\left(D_{2}\right)$ are placed at a shorter distance, creating the "gate" signal for the interference signal processing. This gate is used to trigger and stop/reset a HAMEG-8122 counter, which provides a TTL output for each interference fringe. The gate and the two TTL signals are fed into the acquisition card. A computer code, in our case developed in LabVIEW [11], computes equation 11, providing a wavelength measurement every $1.5 \mathrm{~s}$. Therefore, provided the acquisition card is present, the only hardware modifications needed for the implementation of the numerical method is the incorporation of three BNC cables with respect to the classical configuration based only on the counter itself.

In order to test this method, a diode laser emitting around $845 \mathrm{~nm}$ was used. The laser light was split and sent to the wavemeter and inside a ppKTP crystal for frequency-doubling to reach the $4 s^{2} \rightarrow 4 s 4 p$ transition of Ca. The frequency doubled laser beam cross an 


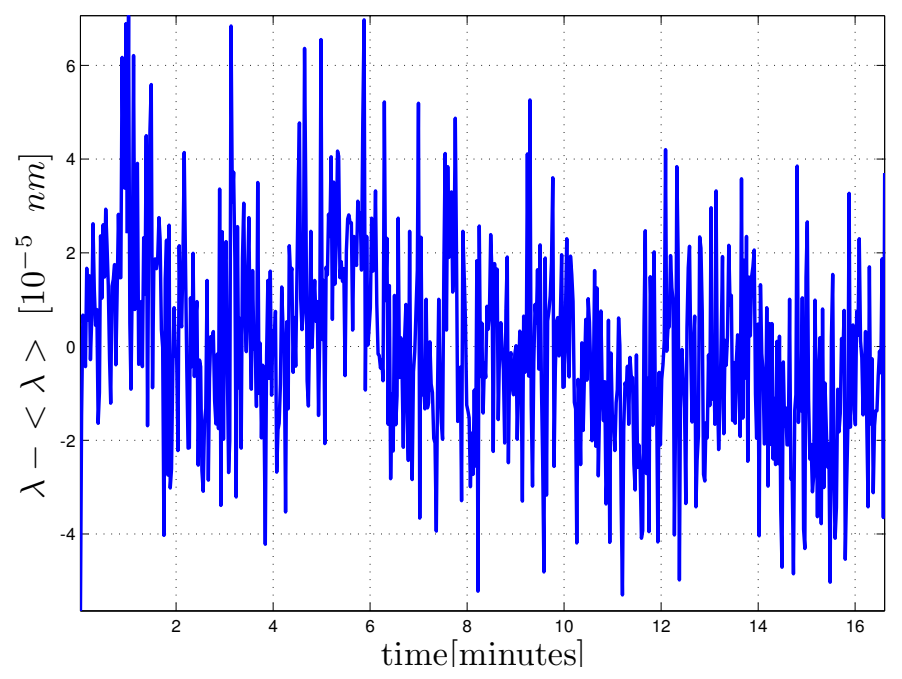

FIG. 4: Time evolution of the wavelength of a laser beam whose first harmonic is locked on the resonance transition of calcium. The wavelength is measured with the described improved method.

atomic beam of neutral calcium in a vacuum chamber in a Doppler-free configuration. The fluorescence signal of the calcium resonance line was monitored by a photomultiplier. The diode laser was locked to the maximum of this fluorescence signal, keeping the $845 \mathrm{~nm}$ laser shortterm frequency fluctuations lower than $3 \mathrm{MHz}$ over the whole duration of the measurement. The evolution of the wavelength measurement residuals $(\lambda-\langle\lambda\rangle)$ is shown in figure 4 . The observed linear drift in figure 4 is probably due to a drift of the lock, while the slow oscillations are correlated to the room temperature variations due to the air conditioning cycle. The He-Ne reference laser was temperature stabilized, providing an estimated stability of $\approx 4 \mathrm{MHz}$. This leads to a theoretical uncertainty of $\left|\frac{\Delta \lambda}{\lambda}\right|_{\text {the }}=2.8 \cdot 10^{-8}$. The data in figure 4 gives $\left|\frac{\Delta \lambda}{\lambda}\right|_{\text {exp }}=2 \sigma_{\lambda} / \bar{\lambda}=5 \cdot 10^{-8}$, which is in very good agreement with the expected value taking into account the measurement uncertainty, the short term fluctuations of the laser frequency and its long term drift.

For comparison, the theoretical uncertainty for one measurement using the apparatus from [1] is $\mid \frac{\Delta \lambda}{\lambda}\left(\left.\right|_{\text {the }}=\right.$ $4.0 \cdot 10^{-8}$ with a measuring time of $20 \mathrm{~s}$ and a clock of $5 \mathrm{MHz}$. After 60 measurements, an experimental uncertainty of $\left|\frac{\Delta \lambda}{\lambda}\right|_{\text {exp }}=2 \sigma_{\lambda} / \bar{\lambda}=2 \cdot 10^{-8}$ is reported, when using as reference and as unknown lasers two He-Neon lasers, both of them locked to the same hyperfine line of molecular iodine-127 [1]. More complex implementations obtain similar results. For example, a vertical version of the wavemeter [13] provides a $\Delta \nu=1.5 \mathrm{MHz}$ with a measuring time of 1 hour, while Kowalski et. al. [9] obtained $\Delta \nu=8 \mathrm{MHz}$ with a measured time of 1 day (the reference does not give more details). By comparing our estimated error with those values, the method proposed here provides an excellent precision with only $1.5 \mathrm{~s}$ of measuring time and very little technical complexity. This is possible by using the fast clock available on the acquisition card. If a slower clock had to be used, the same method proposed will still be applicable, but the speed, $v$, of the carrier would have to be reduced in order to achieve the same performance, thus decreasing the rate at which a measurement is obtained.

\section{CONCLUSION}

A new computer-controlled method has been presented that allows for high resolution measurement by a travelling Michelson wavemeter with a relative simple set-up, possibly improving by two orders of magnitude the initial precision of the apparatus. The simultaneous counting of the number of interference fringes and their time stamp allows a precision of the order of $10 \mathrm{MHz}$ in the nearinfrared domain, when using a $4 \mathrm{MHz} \mathrm{He}-\mathrm{Ne}$ reference laser and a $100 \mathrm{MHz}$ acquisition clock. The simplicity of the method compared with those found in the literature, make it an appealing option when building a new wavemeter. A major advantage of the presented scheme is the possibility to work either in "low" precision mode with a high repetition rate, or in a "high" precision mode with a low measurement rate. Moreover, this technique constitutes a very simple upgrade for an existing Michelson wavemeter whose interference signal is simply processed by a counter.

\section{ACKNOWLEDGEMENTS}

This work is partly funded by CNES under contract no. 116279/00 and by ANR under contract ANR-08-JCJC0053-01. 
[1] S. J. Bennett and P. Gill. A digital interferometer for wavelength measurement. Journal of Physics E: Scientific Instruments, 13(2):174-177, February 1980.

[2] A. Bränberg and P. Nylén. Vacuum-operated digital wavelength meter. Review of Scientific Instruments, 56(2):211, 1985.

[3] C. Champenois, J. Pedregosa-Gutierrez, M. Marciante, D. Guyomarc'h, M. Houssin, M. Knoop AIP Conference Proceedings, 1521, 2013

[4] A. De Marchi. Frequency standards and metrology. Springer-Verlag, 1989.

[5] W. Demtröder. Laser Spectroscopy: Basic Concepts and Instrumentation. Advanced Texts in Physics. Springer, 2003.

[6] J. L. Hall and S. A. Lee. Interferometric real-time display of $\mathrm{cw}$ dye laser wavelength with sub-Doppler accuracy. Applied Physics Letters, 29(6):367, 1976.

[7] J. Ishikawa, N. Ito, and K. Tanaka. Accurate wavelength meter for cw lasers. Applied optics, 25(5):639, March 1986.
[8] A. Kahane. Vernier fringe-counting device for laser wavelength measurements. Review of Scientific Instruments, 54(9):1138, 1983

[9] F. V. Kowalski, R. T. Hawkins, and A. L. Schawlow. Digital wavemeter for $\mathrm{cw}$ lasers. Journal of the Optical Society of America, 66(9):965, September 1976.

[10] F. V. Kowalski, R. E. Teets, W. Demtröder, and A. L. Schawlow. An improved wavemeter for cw lasers. Journal of the Optical Society of America, 68(11):1611, November 1978.

[11] LabVIEW 2011 National Instruments.

[12] J. P. Monchalin, M. J. Kelly, J. E. Thomas, N. A. Kurnit, A. Szöke, F. Zernike, P. H. Lee, and A. Javan. Accurate laser wavelength measurement with a precision twobeam scanning Michelson interferometer. Applied optics, 20(5):736-57, March 1981.

[13] A. Morales, J. de Urquijo, and a. Mendoza. Vertical high-precision Michelson wavemeter. Review of Scientific Instruments, 64(1):76, 1993. 